\title{
Transcatheter pancreatoscopy-guided electrohydraulic lithotripsy for large pancreatic duct stones
}

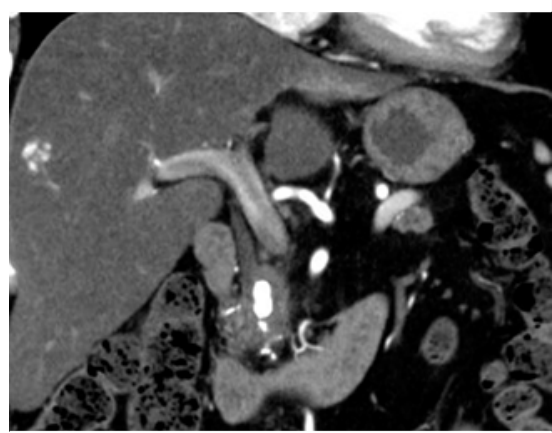

- Fig. 1 Computed tomography showing a large cluster of stones in the main pancreatic duct.

A 61-year-old man was admitted for treatment of alcohol-related chronic pancreatitis with recurrent acute exacerbations. Computed tomography (CT) scanning showed a large cluster of stones in the main pancreatic duct of the head of the pancreas and upstream duct dilatation ( $\triangleright$ Fig. 1). We used endoscopic retrograde cholangiopancreatography (ERCP) and pancreatoscopy with electrohydraulic lithotripsy (EHL) to reduce the patient's stone burden and ductal hypertension. Pancreatoscopy was attempted using the SpyGlass Direct Visualization System with a SpyScope catheter (Boston Scientific, Natick, Massachusetts, USA), but the catheter was too large to pass through the duct without dilation. Therefore, we used a double-lumen catheter (Introducer of a CytoMax II Double Lumen Biliary Cytology Brush; Cook Medical, Winston-Salem, North Carolina, USA), which is smaller than the SpyScope catheter. A SpyGlass optical probe and an EHL probe (Walz Elektronik GmbH, Rohrdorf, Germany) could then be passed together through the lumen of the catheter.

After pancreatic sphincterotomy had been performed, the impacted stones were identified by transcatheter pancreatoscopy, with irrigation being provided through the lumen of the catheter containing the probe ( $\mathbf{F i g . 2}$ ). EHL successfully fragmented most of the impacted stones under direct vision, and a guidewire was successfully passed through the stones ( $\triangleright$ Video 1 ). The fragments were removed with an ERCP basket, and a pancreatogram showed improvement in the upstream duct dilatation ( $>$ Fig. 3 ). There were no procedure-related complications.

Pancreatoscopy-guided EHL is often difficult because of limited control of the field of view, especially when a tortuous and narrow pancreatic duct is involved. Transcatheter endoscopy with an ERCP catheter and Spyglass optical probe has been previously reported to be useful for approaching and observing a narrow portion of the pancreaticobiliary tract, although the technique was used for diagnosis only [1]. Transcatheter pancreatoscopy with EHL using a double-lumen catheter for fragmentation of a large

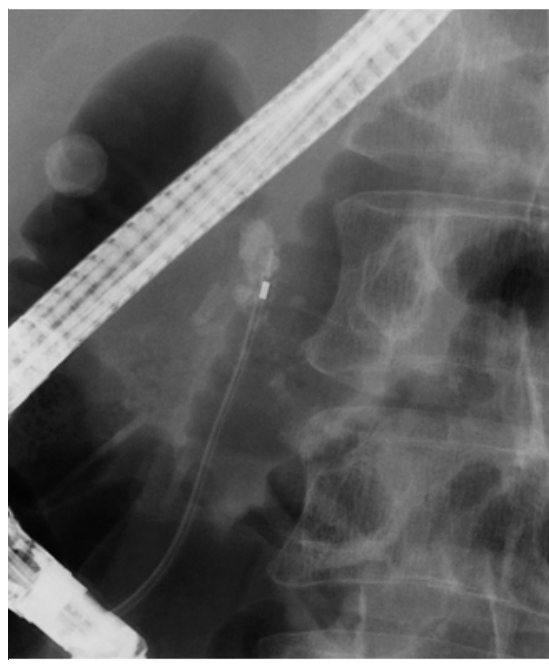

- Fig. 2 Transcatheter pancreatoscopyguided electrohydraulic lithotripsy being performed using a double-lumen catheter with the SpyGlass optical probe.

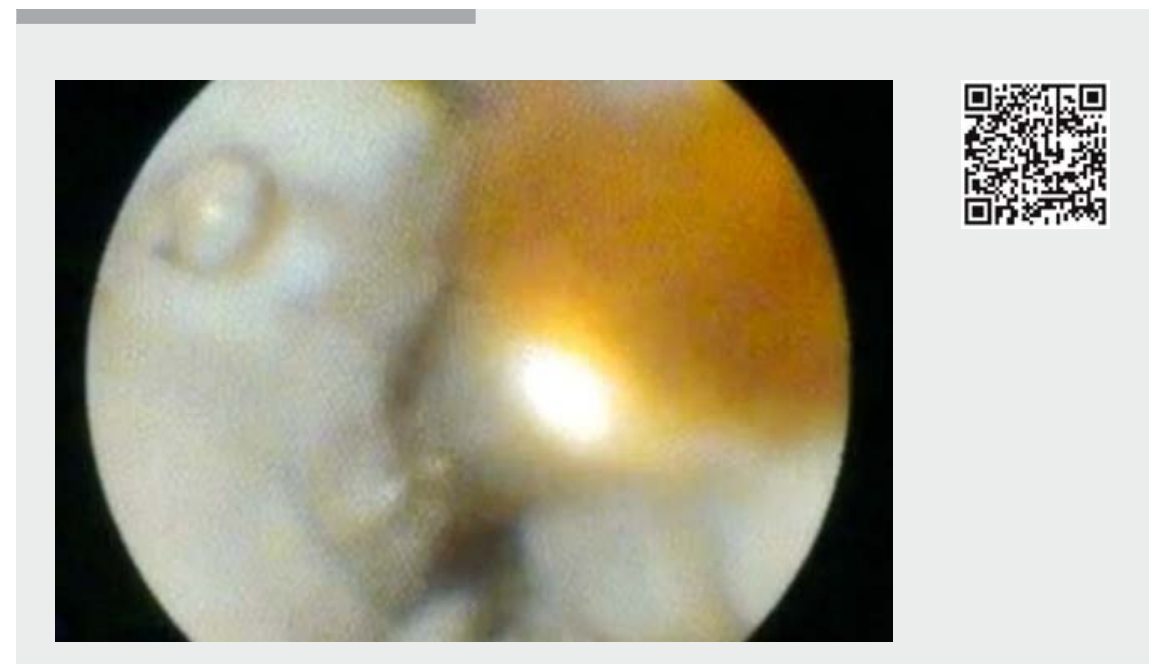

$\checkmark$ Video 1: Fragmentation of stones within the main pancreatic duct using transcatheter pancreatoscopy-guided electrohydraulic lithotripsy under direct vision. 


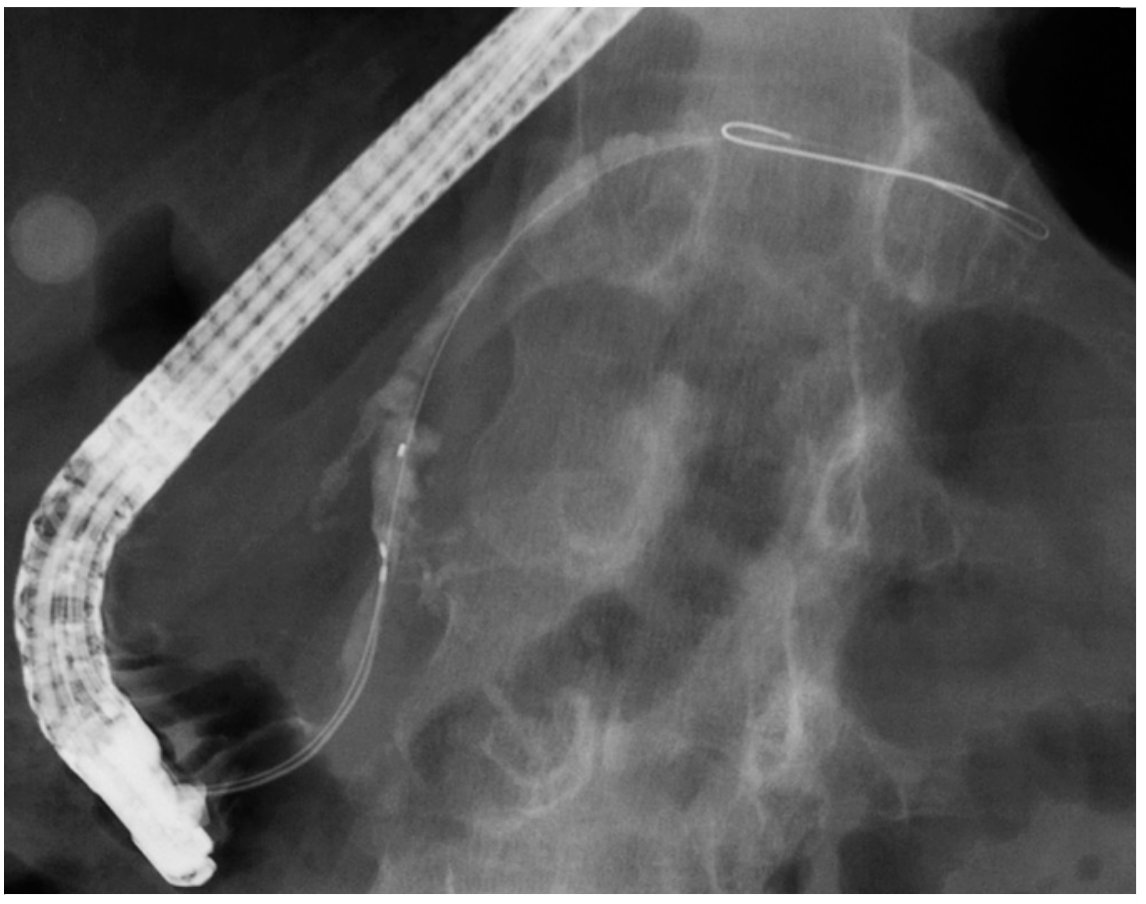

- Fig. 3 Pancreatogram showing improvement of the pancreatic duct dilatation.

cluster of stones and decompression of the duct was performed successfully in our patient without complications.

\section{Competing interests}

None

\section{The Authors}

Yuji Hodo, Yukihiro Shirota, Tsuyoshi Suda, Tokio Wakabayashi

Department of Gastroenterology, Saiseikai

Kanazawa Hospital, Kanazawa, Ishikawa, Japan

\section{Corresponding author}

\section{Yuji Hodo, MD, PhD}

Department of Gastroenterology, Saiseikai Kanazawa Hospital, 13-6 Akatsuchi Machi Ni, Kanazawa, Ishikawa 920-0353, Japan

Fax: +81-76-2661070

y-houdou@saiseikaikanazawa.jp

\section{Reference}

[1] Sakamoto H, Kitano M, Kamata K et al. Transcatheter endoscopy for pancreaticobiliary duct diseases (with videos). Gastrointest Endosc 2012; 76: 892 - 899

\section{Bibliography}

DOI https://doi.org/10.1055/s-0043-105575

Endoscopy 2017; 49: E147-E148

(c) Georg Thieme Verlag KG

Stuttgart · New York

ISSN 0013-726X

Endoscopy_UCTN_Code_TTT_1AR_2AI
This section has its own submission website at

https://mc.manuscriptcentral.com/e-videos 